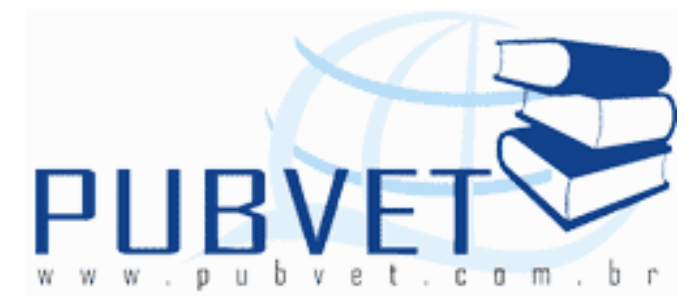

PUBVET, Publicações em Medicina Veterinária e Zootecnia.

Aspectos da utilização de sombreamento em pastagens

Janio Lopes Torquato ${ }^{1}$, Geovan Figueirêdo de Sá Filho ${ }^{1}$, João Batista Freire de Souza Junior ${ }^{1}$, Hérica Girlane Tertulino Domingos ${ }^{1}$, Leonardo Lelis de Macêdo Costa $^{1}$, Maiko Roberto Tavares Dantas ${ }^{1}$

${ }^{1}$ Universidade Federal Rural do Semi-Árido, Departamento de Ciências Animais, Laboratório de Biometeorologia, Biofísica Ambiental e Bem-Estar Animal (LABBEA), BR 110, Km 47, Costa e Silva, CEP: 59625-900, Mossoró-RN.

\title{
Resumo
}

A falta de pesquisas e incentivo para fomentar projetos que apresentem a importância de se manter árvores ou de construir abrigos no pasto para os animais de criação é um dos problemas a serem solucionados na área de produção animal. Ultimamente foram feitos vários trabalhos sobre o ganho de produção em rebanhos, devido ao uso de sombra ou abrigos. Em um trabalho foi constatado um aumento de $20 \%$ na média de produção do gado de leite que tinha acesso a sombra. Em todos os casos a temperatura ambiente elevada associada à alta umidade relativa do ar, bem como a radiação solar e outros fatores ambientais, são considerados componentes climáticos estressantes para o rebanho bovino leiteiro, de modo a reduzirem o desempenho produtivo destes animais, uma vez, que vacas em lactação, principalmente as de alta produção, são sensíveis ao estresse térmico. Em diversas regiões do planeta há uma carência de árvores para fornecer uma 
TORQUATO, J.L. et al. Aspectos da utilização de sombreamento em pastagens. PUBVET, Londrina, V. 6, N. 14, Ed. 201, Art. 1348, 2012.

sombra adequada para os animais que são criados no pasto, nestes casos o melhor a se fazer é usufruir de sombreamento artificial, seja móvel ou fixo, no qual é importante para o bem estar animal e melhorar o seu desempenho reprodutivo. Uns pesquisadores observaram o efeito da sombra proporcionada por piquetes em vacas lactantes e concluíram que as vacas com acesso a sombra apresentaram maior produção de leite diária total do que as que não se beneficiaram com sombra. Portanto, é importante de se manter as árvores e construir abrigos para que os rebanhos de criação possam suportar as altas incidencias de raios solares e melhorarem o seu desempenho reprodutivo.

Palavras-chave: sombreamento natural, sombreamento artificial, produção animal

\section{Aspects of the use of shading in grasslands}

\section{Abstract}

The lack of incentives to promote research and projects that show the importance of maintaining trees or build shelters in the pasture for livestock is a problem to be solved in the area of animal production. Recently several studies were made on the gain in livestock production due to the use of shade or shelter. In one study it was found a $20 \%$ increase in average production of dairy cattle that had access to shade. In all cases the high temperature associated with a high relative humidity, as well as the solar radiation and other environmental factors, components are considered too stressful climatic dairy cattle in order to reduce the growth performance of animals, since, that dairy cows, especially those of high production, are sensitive to heat stress. Various regions of the planet there is a lack of trees to provide adequate shade for the animals that are raised on pasture, in these cases the best thing to do is enjoy of shading, whether mobile or fixed, which is important for animal welfare and improve their reproductive performance. Some researchers observed the effect of shade provided by pickets in lactating dairy cows and concluded that cows with access to shade had higher total daily milk 
TORQUATO, J.L. et al. Aspectos da utilização de sombreamento em pastagens. PUBVET, Londrina, V. 6, N. 14, Ed. 201, Art. 1348, 2012.

production than those who did not benefit from shade. Therefore, it is important to keep the trees and build shelters for the flocks of creation can withstand the high incidences of sunlight and improve their reproductive performance.

Keywords: natural shade, artificial shade, animal production

\section{INTRODUÇÃO}

Atualmente no Brasil a grande superfície territorial não é utilizada de forma adequada em relação às pastagens nas propriedades rurais. Mas se for transformada em pastagens arborizadas, ela pode desempenhar uma função importante melhorando o conceito da pecuária brasileira, dessa forma beneficiará a produção animal e a produção de madeira. É um novo desafio para a bovinocultura de o país implantar árvores na criação de rebanhos no pasto. Com a arborização irá aumentar a produção de madeira e serviços ambientais e consequentemente produção animal e, desta forma ajudará uma grande quantidade de produtores rurais que tem na bovinocultura a sua atividade de sustento (PORFÍRIO-DA-SILVA, 2005).

As árvores são pouco utilizadas e não é dada uma devida importância a elas nas propriedades rurais. A arborização das pastagens permite aumentar a área de sombreamento nas áreas de pastagens a céu aberto, para proteger o rebanho de altas temperaturas e ainda obter serviços ambientais e diversificação de produtos florestais e pecuários e, a sombra, também, reduz o estresse térmico dos animais, auxilia no ganho produtivo dos animais (MONTOYA et al., 1994).

Nos dias de intenso calor, nas horas mais quentes, os animais procuram reduzir os efeitos da radiação solar direta e altas temperaturas do ar, abrigando-se na sombra das árvores; aproveitam tais períodos para descansar, ruminar, ou mesmo pastejar, desde que nesses locais haja disponibilidade de forragem. Com a introdução do gado europeu, a pecuárias de corte e de leite nos trópicos, face à grande sensibilidade destes bovinos ao calor, torna-se 
TORQUATO, J.L. et al. Aspectos da utilização de sombreamento em pastagens. PUBVET, Londrina, V. 6, N. 14, Ed. 201, Art. 1348, 2012.

ainda mais importante a existência de áreas sombreadas nas pastagens (ENCARNAÇÃO \& KOLLER, 1997).

A presença de sombras no campo é importante para o conforto dos animais, principalmente em regiões quentes e com alta incidência de radiação solar, nas quais os bovinos da maioria das raças tendem a sofrer estresse térmico, reduzindo o seu desempenho econômico (SILVA, 2006). Com a implantação de árvores no pasto elas podem, também, trazer benéficos serviços ambientais para o produtor como produzir madeira e outros bens florestais (resinas, produtos medicinais), combater a salinidade e problemas de alagamento, proteger e conservar os solos, prover sombra e abrigo para outras plantas e animais, conserva e encoraja a biodiversidade, melhora a beleza do local (ABEL et al., 1997).

Os estudos de avaliação da qualidade da sombra natural ou artificial têm como o objetivo de aumentar a produção, assim fornecendo ao animal um ambiente em que ele possa expressar todo o seu potencial genético e reprodutivo (SILVA et al., 1996).

\section{SOMBREAMENTO NATURAL}

Alguns trabalhos sobre o ganho de produção em rebanhos, devido ao uso de sombra natural, já foram realizados. Por exemplo, o trabalho de HAHN (1981), citado por GHELFI FILHO et al. (1996), onde constatou-se um aumento de $20 \%$ na média de produção do gado de leite que tinha acesso a sombra. Segundo RIVERO (1986), a vegetação absorve, aproximadamente, $90 \%$ da radiação visível e $60 \%$ da infravermelha; o que sobre é transmitido por entre as folhas ou refletido.

Com a utilização da arborização, utilizando Eucaliptus saligna, SILVA et al. (1998b), constatam que a produção de ovos do aviário na região arborizada foi $23,1 \%$ superior, em comparação com a produção de ovos no aviário sem a arborização. Elas proporcionam abrigo contra a radiação solar e ventos excessivos, contribuindo, com sua sombra, na redução da temperatura 
TORQUATO, J.L. et al. Aspectos da utilização de sombreamento em pastagens. PUBVET, Londrina, V. 6, N. 14, Ed. 201, Art. 1348, 2012.

ambiente, pela grande quantidade de água transpirada por suas folhas (CRESTANA, 1994). De acordo com Sánchez (2001), a admissão de árvores e arbustos de uso diverso ou forrageiro em pastagens pode aumentar a quantidade e melhorar a qualidade da forrageira disponível aos animais.

A temperatura ambiente elevada associada à alta umidade relativa do ar, bem como a radiação solar e outros fatores ambientais, são considerados componentes climáticos estressantes para o rebanho bovino leiteiro, de modo a reduzirem o desempenho produtivo destes animais, uma vez, que vacas em lactação, principalmente as de alta produção, são sensíveis ao estresse térmico. (MEDEIROS; VIEIRA, 1997).

Em outro trabalho, SILVA et al. (1996), afirmam que "a importância do sombreamento natural vem sendo discutida cada vez mais no meio acadêmico e científico. A sombra definida como proteção contra a radiação térmica muda o balanço de radiação animal. Uma estrutura de sombra simples pode efetivamente reduzir a carga de calor radiante que incide sobre um animal em $30 \%$ ou mais, pela interceptação da radiação solar direta".

Em estudo realizado por MULLER (1982), citado em BAGGIO E CARPANEZZI (1988), o aumento do ganho de peso em gado de corte, em áreas que a arborização estava presente, ou seja, 1,29 Kg/dia, em contraste com 0,5 Kg/dia, em pastagem a céu aberto, na Califórnia. Já KRATZER (1956) comprova que as áreas desprovidas de vegetação tornam-se muitos quentes ao meio dia e frias à noite, enquanto áreas densamente vegetadas apresentam uma temperatura sem grandes variações diuturnas, sendo, consideravelmente mais frescas que as anteriores.

Várias pesquisas mostram que vacas tendo acesso a sombras apropriadas ocasionam uma melhora na produção de leite (ROMAN-PONCE et al. 1977; INGRAHAM et al. 1979; BUFFINGTON et al. 1983; IGONO, 1986; SILVER, 1987; DAVISON et al. 1988). O mesmo foi observado na Florida onde as vacas foram expostas à intensa radiação solar em um local quente causou a diminuição de 1,5 a 3,3 kg por vaca na produção diária de leite de vacas Holandesas (HANSEN, 1990). 
TORQUATO, J.L. et al. Aspectos da utilização de sombreamento em pastagens. PUBVET, Londrina, V. 6, N. 14, Ed. 201, Art. 1348, 2012.

SALLA et al. (2009) submeteram novilhas leiteiras Holandesas $x$ Zebu ao pastejo rotacionado em piquetes de braquiária providos de sombreamento natural, as que tiveram acesso a sombra obtiveram uma melhor condição de conforto térmico, comparado às novilhas contidas em piquetes desprovidos de sombra, isso foi devido eles observarem que as novilhas que estavam a sombra estiveram mais próximas das suas condições fisiológicas (frequência respiratória, taxa de sudação e temperatura de superfície corporal) normais aceitáveis para a espécie bovina.

SILVA et al (1998a) estudaram o efeito da sombra natural na redução da carga térmica de radiação. Utilizaram três espécies diferentes: Sibipiruna (Caesaelpinia peltophoroides Benth.), Sapucaia (Lecythis pisonis Camb.) e Tipuana (Tipuana speciosa Benth.), constataram que a melhor sombra foi a da Sibipiruna e as demais Tipuana e Sapucaia. Eles ainda compararam as árvores com um galpão coberto com telhas de fibrocimento e a radiação sem sombra, na qual verificaram uma redução da carga térmica de $37,7 \%$ para a sombra natural e $34,4 \%$ pelo telhado de fibrocimento, concluindo que a sombra das arvores é de melhor qualidade que a artificial.

Para TITTO et al. (2008) a disponibilidade de sombra para os animais de produção melhora suas condições fisiológicas (frequência respiratória, temperatura retal, batimentos cardíacos, etc.), no comportamento animal (consumo, ócio, ruminação, etc.) e no desempenho produtivo (carne, leite, etc.), percebendo-se diferenças mais acentuadas nestas variáveis quanto menor for a tolerância dos animais às elevadas temperaturas.

De acordo com BAÊTA E SOUZA (1997); CONCEIÇÃO (2008) animais de produção expostos aos efeitos da radiação direta do Sol sofrem mais com o ganho excessivo de calor do que àqueles animais protegidos em locais sombreados, nas quais as sombras de árvores possibilitam um maior conforto animal frente às sombras artificiais (tela sombrite, telha cerâmica, amianto, metal galvanizado, etc.), sendo, o provimento de sombras em uma pastagem animal a forma mais econômica de proporcionar conforto e bem estar térmico animal. 
TORQUATO, J.L. et al. Aspectos da utilização de sombreamento em pastagens. PUBVET, Londrina, V. 6, N. 14, Ed. 201, Art. 1348, 2012.

MAGALHÃES et al. (1998) em sistemas silvipastoris, com seringueiras na pastagem de $B$. brizantha, compararam novilhos bubalinos a sombra e expostos ao sol, os novilhos a sombra mostraram media de ganho de 0,757 e $0,831 \mathrm{~kg} /$ animal/ dia nas estações secas e chuvosas, respectivamente, em relação aos que estavam a pleno sol, onde obtiveram media de ganhos de peso diário em torno de 0,337 kg.

\section{SOMBREAMENTO ARTIFICIAL}

Existe no mundo regiões que não possui muita disponibilidade de árvores para fornecer uma sombra adequada para os animais que são criados no pasto, nestes casos o melhor a se fazer é usufruir de sombreamento artificial, seja móvel ou fixo, no qual é importante para o bem - estar animal e melhorar o seu desempenho reprodutivo.

Estudo feito por MITLOHNER (2001) ele afirma que o estresse calórico impactou negativamente na produção dos novilhos confinados no Texas. Os novilhos mantidos a sombra mostraram maior peso de carcaça que os novilhos expostos ao sol. Com um resultado econômico de US\$18,00 a favor dos animais mantidos em confinamento com acesso à sombra artificial.

CHIQUITELLI NETO (2005) trabalhando com novilhas da raça nelore em confinamento, com sombra artificial (SA) e sem sombra (SS), não encontrou diferença na taxa de ganho de peso, no entanto, os animais do lote SA apresentaram maior índice de gordura de cobertura com valor médio de 3,45 pontos contra 3,25 do grupo SS. Além disso, encontrou diferença da quantidade animais dentro dos níveis de classificação em grau de acabamento, favorável comercialmente para o grupo AS $(P<0,05)$, mostrando a influência da sombra sobre a melhoria na eficiência na utilização dos nutrientes proveniente da alimentação pelo balanço energético positivo, proporcionado pela sombra na homeostase.

Outros pesquisadores relataram que vacas preferem sombra natural em vez de estruturas artificiais devido à eficiência no bloqueio de radiação solar e 
TORQUATO, J.L. et al. Aspectos da utilização de sombreamento em pastagens. PUBVET, Londrina, V. 6, N. 14, Ed. 201, Art. 1348, 2012.

a evaporação das folhas, que resfriam o ar ambiente (por exemplo, SHEARER et al., 1991). As árvores, no entanto, pode ter um curto período de vida em pastagens para os rebanhos leiteiros grande (SHEARER et al., 1991) e, portanto, a prestação de outros tipos de sombra pode ser mais prático.

Já um estudo feito por LEVA \& GALLARDO \& VALTORTA (1997) na qual eles utilizaram para sombrear os animais um tecido preto de propileno, proporcionando $80 \%$ de sombra, colocados a $2,5 \mathrm{~m}$ de altura em eucaliptus. Constataram que a media de temperatura retal nas vacas que estavam na sombra foi de $39.3{ }^{\circ} \mathrm{C}$ (SD 0,42), e $40.1{ }^{\circ} \mathrm{C}$ (SD 0,74) para o grupo nãoprotegido, enquanto taxa respiratória média foi de 60,7 (SD 10,57) e 78,9 (SD $18,04)$, respectivamente, ambas as diferenças sendo significativas $(P<0,01)$.

SCHUTZ et al. (2009) avaliaram o comportamento de vacas leiteiras holandesas em clima temperado, e quando os animais foram submetidos a combinações de oferta de níveis diferentes de proteção de sombra artificial, constituídas por tecidos específicos com 99 e 25; 99 e 50 e 50 e $25 \%$ de proteção contra a radiação solar, verificaram que nas horas mais quentes dos dias pesquisados, $73,3 \%$ dos animais passaram mais tempo sob a proteção 99 $\%$ na combinação 99 e $25 \%$ ( $P<0,01)$, e $72 \%$ dos mesmos preferiram a proteção de $50 \%$ na combinação 50 e $25 \%(P<0,01)$. Eles verificaram que o maior efeito positivo da qualidade do sombreamento e evidenciado nos horários em que a radiação solar e mais elevada, ou seja, nos horários mais quentes do dia (10:00 às $15 \mathrm{~h}: 00 \mathrm{~min}$ ), em que foram observadas reduções percentuais medias de carga térmica radiante (CTR) de 12,$3 ; 9,6$ e 7,2 \% $(P<0,05)$ para as telhas de fibrocimento, galvanizadas e tela de polipropileno, respectivamente, em relação ao tratamento controle (sem sombra).

MELLACE (2009), trabalhando com efeito do tamanho de area de cobertura de abrigos, sobre o conforto de novilhas Jersey e Holandesas puras, e ainda, mestiças de ambas as raças, verificou diferença estatística $(P<0,05)$ na temperatura de globo negro (TGN) entre uma area sem sombra (controle), comparada as áreas com 1,$5 ; 3,0 ; 5,0$ e 8,0 m2 de sombra animal-1, 
TORQUATO, J.L. et al. Aspectos da utilização de sombreamento em pastagens. PUBVET, Londrina, V. 6, N. 14, Ed. 201, Art. 1348, 2012.

constatando uma redução desta variável de 37,2 para $29,8{ }^{\circ} \mathrm{C}$, da area não sombreada frente à de $8,0 \mathrm{~m} 2$ animal-1, representando um decréscimo de 7,4 ${ }^{\circ} \mathrm{C}(19,89 \%)$.

Em um estudo feito por MITLOHNER, GALYEAN \& MCGLONE (2002) constataram que as novilhas que foram submetidas à sombra de um abrigo feito de alumínio galvanizado ou revestido de zinco obtiveram menor taxa de respiração do que os as novilhas que não tiveram acesso ao abrigo.

KENDALL et al. (2006) observaram o efeito da sombra proporcionada por piquetes em vacas lactantes e concluíram que as vacas com acesso a sombra apresentaram maior produção de leite diária total do que as que não se beneficiaram com sombra. Isso foi evidente durante a ordenha da manhã

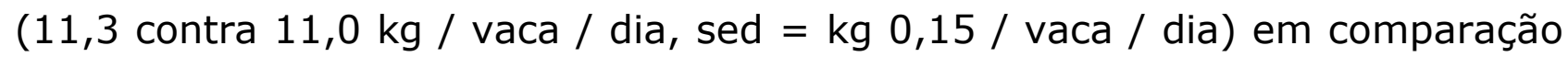
com a ordenha da tarde $(6,4$ contra $6,2 \mathrm{~kg} /$ vaca / dia, sed $=0,08 \mathrm{~kg} /$ vaca / dia).

NAAS e ARCARO Jr. (2001). Estes autores avaliando a influencia da sombra artificial (S), sombra com ventilação (SV) e sombra com ventilação e adição de aspersão (SVA) sobre o desempenho produtivo de vacas leiteiras de alta produção, verificaram, na ordenha da manha, melhor desempenho produtivo lácteo para o SVA, seguido do SV e por ultimo o $S(P<0,05)$, enquanto à tarde, embora o melhor resultado tenha sido também observado para o SVA $(P<0,05)$, não foi identificado, diferença estatística entre SV e S neste turno. Quando observada a produção total diária obtida no experimento de Naas e Arcaro Jr. (2001), foi registrada uma diferença de 2,33 kg de leite animal dia-1 (11,33 \%) entre SVA e S.

EIGENBERG et al. (2007) avaliaram diferentes tipos de materiais para fornecer sombreamento artificial e concluíram que todos eles apresentaram melhores condições de bem-estar aos animais em comparação com um local sem nenhuma proteção contra a radiação solar direta e as altas temperaturas. 
TORQUATO, J.L. et al. Aspectos da utilização de sombreamento em pastagens. PUBVET, Londrina, V. 6, N. 14, Ed. 201, Art. 1348, 2012.

\section{CONSIDERAÇÕES FINAIS}

Pode-se observar que o sombreamento das pastagens com sombras artificiais e/ou naturais é essencial para melhorar a produção animal, principalmente para as regiões em que predomina o clima quente com altas incidências de radiação solar. Sem duvida a melhor sombra é a oferecida pelas árvores, mas devido a alguns atributos (solo, nutrientes, clima e tempo) que elas precisam para serem cultivadas. Assim, os abrigos artificiais é uma solução ótima e imediata para o problema da ausência de sombra para os animais no pasto nas horas mais quentes do dia.

\section{REFERÊNCIAS}

ABEL, N.; BAXTER, J.; CAMPBELL, A. et al. Design Principles for Farm Forestry: A guide to assist farmers to decide where to place trees and farm plantations on farms.

RIRDC/LWRRRRDC/FWPRDC Joint Venture Agroforestry Program, 1997.

BAÊTA, F. C.; SOUZA, C. F. Ambiência em edificações rurais: conforto animal. Viçosa: UFV, 246p, 1997.

BAGGIO, A. J.; CARPANEZZI, 0. B. Alguns sistemas de arborização de pastagens. Curitiba: Boletim Pesquisa Florestal, (17), 39-46, dez. 1988.

BUFFINGTON, D.E.; COLLIER, R.J.; CANTON, G.H. Shade management systems to reduce heat stress for dairy cows in hot, humid climates. Transactions of the ASAE, St. Joseph, v.26, p.1798-802, 1983.

CHIQUITELLI NETO, M. Sombreamento artificial como tecnologia para melhorar a eficiência econômica e o bem estar na produção de bovinos de corte. 2005. 77 p. Tese (Doutorado em Zootecnia) - Faculdade de Ciencias Agrarias e Veterinarias, Universidade Estadual Paulista Júlio de Mesquita Filho, Jaboticabal, 2005.

CONCEICAO, M. N. Avaliação da influência do sombreamento artificial no desenvolvimento de novilhas em pastagens. 2008. 137f. Tese (Doutorado em Agronomia) - Universidade de Sao Paulo - Escola Superior de Agricultura "Luiz d Queiroz", Piracicaba, 2008.

CONCEIÇÃO, M. N. Avaliação da influência do sombreamento artificial no desenvolvimento de novilhas em pastagens. 2008. 137f. Tese (Doutorado em Agronomia) - Universidade de São Paulo - Escola Superior de Agricultura "Luiz d Queiroz", Piracicaba, 2008.

CRESTANA, M. S. M.; ERBOLATO JUNIOR, N. Arborização adequada de cidades (Curso).

Campinas: Apostila do curso, p. 38, 1994. 
DAVISON, T.M.; SILVER, B.A.; LISLE, A.T.; ORR, W.N. The influence of shade on milk production of Holstein-Friesian cows in a tropical upland environment. Australian Journal of Experimental Agriculture, Melbourne, v.28, p.149-54, 1988.

EIGENBERG, R.A.; BROWN-BRANDL, T. M.; NIENABER, J.A. Shade material evaluation using a cattle response model. Disponível em:

http: //asae.frymulti.com/azdez.asp?JID $=5 \& A I D=24040 \& C I D-\min 2007 \& T=2$ Acesso em: 05 abr. 2009.

ENCARNAÇÃO, R. de O.; KOLLER, W.W. A importância de bosques nas pastagens. Informe Agropecuario, Campo Grande, Sindicato Rural de Campo Grande, 10 (141): 12, Nov/1997.

HAHN, L.G. Housing and management to reduce climatic impacts on livestock Journal of Animal Science, Menasha, v. 52, n. 1, p. 175-186, 1981. Apud GHELFI FILHO, Hugo et a!. Sombreamento natural: avaliaviio de algumas especies arb6reas atraves de indices de conforto termico. In: CONGRESSO BRASILEIRO DE ENGENHARIA AGRiCOLA, 25, 1996, Bauru. Anais. Bauru: SBEA, 1996. paper n.395-96. 8 p.

HANSEN, P.J. Effects of coat colour on physiological responses to solar radiation in Holsteins. Veterinary Record, London, v.127, p.333-4, 1990.

IGONO, M.O. Effect of a humid temperate climate and environmental modifications with shade, spray and fan, on milk production, thermal balance and hormone function of dairy cows. Dissertation Abstracts International. Section B, Sciences and Engineering, Ann Arbor, v.46, p.3645, 1986.

INGRAHAM, R.H.; STANLEY, R.W.; WAGNER, W.C. Seasonal effects of tropical climate on shaded and non-shaded cows as measured by rectal temperature, adrenal cortex hormones, thyroid hormone, and milk production. American Journal of Veterinary Research, Chicago, v.40, p.1792-7, 1979.

KENDALL, P. E; NIELSEN, P. P; WEBSTER, J. R; VERKERK, G. A; LITTLEJOHN, R. P.; MATTHEWS, L. R. The effects of providing shade to lactating dairy cows in a temperate climate. Livestock Science, v.103, p. 148-157, 2006.

KRATZER, P. B. The climate of cities. Braunchweig: vieweg and sohn, 837p, 1856.

LEVA, P. E.; VALTORTA, S. E.; GALLARDO, M. R. Evaluation of different shades to improve dairy cattle well-being in Argentina. Int J Biometeorol, 41, p.65-67, 1997.

MAGALHÃES, J.A.; TAKIGAWA, R.M.; TAVARES, A.C. et al. Tolerância de bovídeos a temperatura e umidade do trópico úmido. Porto Velho: EMBRAPA-CPAF Rondônia, 4 p. (EMBRAPA-CPAF Rondônia. Comunicado Técnico, 147), 1998.

MELLACE, M. E. Eficiência da área de sombreamento artificial no bem-estar animal de novilhas leiteiras criadas a pasto. 2009. 95f. Dissertacao (Mestrado em Agronomia) - Universidade de São Paulo - Escola Superior de Agricultura "Luiz d Queiroz", Piracicaba, 2009.

MITLOHNER, F. M; GALYEAN, M. L; MCGLONE, J. J. Shade effects on performance, carcass traits, physiology, and behavior of heat-stressed feedlot heifers. Journal Animal Science, v. 80, p. 2043-2050, 2002.

MONTOYA, L. J.; MEDRADO, M. J. S.; MASCHIO. L.M. DE A. Aspectos de arborização de pastagens e viabilidade tecnica-economica da alternativa silvipastoril . In. SEMINARIO SOBRE 
SISTEMAS AGROFLORESTAIS NA REGIAO SUL DO BRASIL, 1., Colombo. Colombo: EmbrapaCNPF, p. p.157-172, 1994.

MÜLLER, P. B. Bioclimatologia aplicada aos animais domésticos. Porto Alegre: Sulina. p. 158, 1982.

NAAS, I. A.; ARCARO Jr, I. Influencia de ventilacao e aspersao em sistemas de sombreamento artificial para vacas em lactacao em condicoes de calor. Rev. Brasileira de Engenharia Agrícola e Ambiental, v.5, n.1, p.139-142, 2001.

PORFÍRIO-DA-SILVA. Arborização de pastagens como prática de manejo ambiental e estratégia para o desenvolvimento sustentável do Brasil pecuário. In: CONGRESSO

BRASILEIRO DE RAÇAS ZEBUÍNAS, 6, Uberaba, MG, 2005. Palestras... Uberaba, MG, 2005.

RIVERO, R. Arquitetura e clima. Acondicionamento térmico natural. Porto Alegre: D. C. Luzzatto, 1986.

ROMAN-PONCE, H.; THATCHER, W.W.; BUFFINGTON, D.E.; WILCOX, C.J.; HORN, H.H. Physiological and production responses of dairy cattle to a shade structure in a subtropical environment. Journal of Dairy Science, Champaign, v.60. p.424-31, 1977.

SALLA, L.; PIRES, M. F. A.; MORAIS, D.; DIAS, M.; OLIVEIRA, P.; SANTOS, B. C. Efeito da disponibilidade de sombra sobre o conforto térmico de novilhas leiteiras. Rev. Brasileira de Agroecologia, v. 4 n. 2, p. 3343-3346, 2009.

SÁNCHEZ, M.D. Panorama dos sistemas agrofrorestais pecuários na América Latina. In: CARVALHO, M.M.; ALVIM, M.J.; CARNEIRO, J.C. (Eds). Sistemas agroflorestais pecuários: opções de sustentabilidade para áreas tropicais e subtropicais. Juiz de Fora: Embrapa Gado de Leite, p.9-17, 2001.

SCHUTZ, K. E.; ROGERS, A. R.; COX, N. R. TURCKER, C. B. Dairy cows prefer shade that offers greater protection against solar radiation in summer: shade use, behavior, and body temperature. Rev. Applied Animal Behaviour Science, v. 116, p. 28-34, 2009.

SHEARER, J.K., BEEDE, D.K., BUCKLIN, R.A., BRAY, D.R. Environmental modifications to reduce heat stress in dairy cattle. Agri-Practice12,7-18, 1991.

SILVA, I. J. O. GHELFI FILHO, H.; NÄÄS, I. A. ; GARBOGGINI, I. L. A. Redução da carga térmica de radiação através do sombreamento natural. IN: CONGRESSO DE INGENIERIA RURAL, $4^{\circ}$, CONGRESSO INTERNACIONAL DE INGENIERIA RURAL, $2^{\circ}$, 1996. Neuquen.

Memorias... Neuquen: Universidad Nacional del Comahe, Universidad Nacional de La Plata, INTA Centro Regional Patagonia Norte, p. 799-807, 1996.

SILVA, I.J.O.; SILVA, K.O.; NÄÄS, I.A. Arborização: uma metotologia para avaliação da qualidade de sombras por meio dos indices de conforto térmico, In: II Congresso Brasileiro de Biometeorologia, Anais...Goiânia- GO, 1998a.

SILVA, R. G. PREDIÇÃO DA CONFIGURAÇÃO DE SOMBRAS DE ÁRVORES EM PASTAGENS PARA BOVINOS. Eng. Agríc., Jaboticabal, v.26, n.1, p.268-281, jan./abr. 2006.

SILVA. I. J. O.; NÄÄS, I. A.; MENEZES, J. F. Uso da arborização e desempenho térmico de aviários através dos índices de conforto térmico e produção de ovos. IN: CONGRESSO BRASILEIRO DE ENGENHARIA AGRÍCOLA, 27²,1998b, Poços de Caldas. Anais... Poços de Caldas: Sociedade Brasileira de Engenharia Agrícola, p. 231-233, 1998b. 
SILVER, B.A. Shade is important for milk production. Queensland Agricultural Journal, Brisbane, v.113, p.95-6, 1987.

TITTO, E. A. L.; PEREIRA, A. M. F.; VILELA, R. A.; TITTO, C. G.; AMADEU, C. C. B. Manejo ambiental e instalações para vacas leiteiras em ambiente tropical. In: WORKSHOP DE AMBIÊNCIA NA PRODUÇÃO DE LEITE, I, 2008, Nova Odessa. Palestras. Nova Odessa: Centro Apta - Bovinos de Leite do Instituto de Zootecnia, p.1-24, 2008. 